\title{
Spatial working memory activity of the caudate nucleus is sensitive to frame of reference
}

\author{
BRADLEY R. POSTLE \\ University of Wisconsin, Madison, Wisconsin \\ and \\ MARK D'ESPOSITO \\ University of California, Berkeley, California
}

\begin{abstract}
We used event-relatedfMRI to test the hypothesis that the caudate nucleus is preferentially recruited by a spatial working memory task employing egocentrically defined stimuli, which are amenable to transformation into a motor code, as contrasted with allocentrically defined stimuli, which are not. Our results revealed greater delay-epoch activity in egocentric than in allocentric trials in the caudate nucleus and trends in the same direction in the putamen and the lateral premotor cortex (PMC). Response-related activity was greater for egocentric trials in the lateral PMC. We propose that the neostriatum, possibly interacting with the PMC, may contribute to the sensory-motor transformation necessary to establish a prospective motor code (e.g., the representation of a saccade or a grasp). In addition, the PMC may participate in decision-making processes, prompted by the onset of the probe stimulus, that employ this prospective motor information. This model accounts for the empirical evidence that motor distraction disrupts spatial working memory performance.
\end{abstract}

Data and principles from neuroscience have imposed constraints on models of the cognitive architecture of working memory. For example, the idea that visual working memory for different domains of information is computed by partially independent systems (Della Sala, Gray, Baddeley, Allamano, \& Wilson, 1999; Logie, 1995; Smith et al., 1995; Tresch, Sinnamon, \& Seamon, 1993) originated with the proposal that the neuroanatomical and neurophysiological organizational principles of the primate visual system (Ungerleider \& Haxby, 1994; Ungerleider $\&$ Mishkin, 1982) might extend to visual working memory (Goldman-Rakic, 1987, 1990). Recent neuroimaging studies of visual working memory have focused primarily on the systems neuroscience question of specifying the cortical systems that support spatial and object working memory (e.g., Courtney, Ungerleider, Keil, \& Haxby, 1996; D’Esposito, Aguirre, Zarahn, \& Ballard, 1998; Postle, Stern, Rosen, \& Corkin, 2000; Smith et al., 1995). However, neuropsychological evidence of impaired visuospatial working memory in monkeys with caudate nucleus lesions

This research was supported by the American Federation for Aging Research and by NIH Grants NS01762 and AG13483. B.R.P. also received support from NIH Grant AG00255 (to Virginia M.-Y. Lee, University of Pennsylvania Medical Center). We thank Maria Alavanja, Jeffrey Berger, Olufunsho Faseyitan, and John Koger for assistance with experiment development, programming, scanning, and data management and Anjan Chatterjee, David Meyer, and Eric Zarahn for helpful discussions of this work. Correspondence concerning this article should be addressed to B. R. Postle, Department of Psychology, University of Wisconsin, 1202 West Johnson St., Madison, WI53706(e-mail: postle@ wisc.edu).
(Battig, Rosvold, \& Mishkin, 1960; Dean \& Davis, 1959; Divac, Rosvold, \& Szwarcbart, 1967; Goldman \& Rosvold, 1972; Rosvold \& Delgado, 1956) and of a selective impairment of spatial, but not object, visual working memory in patients in the early stages of Parkinson's disease (PD; Owen, Iddon, Hodges, Summers, \& Robbins, 1997; Postle, Jonides, Smith, Corkin, \& Growdon, 1997; Postle, Locascio, Corkin, \& Growdon, 1997) has indicated that the neostriatum may also be an area that underlies the functional segregation of visual working memory. We have begun to explore the implications of these neuropsychological results for models of visual working memory with event-related fMRI studies of basal ganglia activity in healthy participants.

In one previous fMRI study of delayed recognition, we observed that spatial working memory activity was greater in the caudate nucleus when it immediately preceded a motor response than when no overt response followed the delay epoch, a contingent relation not observed with nonspatial memoranda (Postle \& D'Esposito, 1999a, 1999b). In a second experiment, caudate nucleus activity was greater during the first portion of a delay epoch on trials in which the target stimulus predicted the response (Postle \& D'Esposito, 1999a), an effect consistent with prospective coding of the motor response. (We found no reliable memoryrelated activity in the putamen in either of these studies [unpublished observations].) These patterns of caudate nucleus activity could not be explained as anotherinstance of the bifurcation in the brain of the representation of what and where sensory components of a memorandum, because in neither study was there a significant difference between 
spatial and nonspatial delay-related activity in the caudate nucleus when the data were collapsed across delay epochs (Postle \& D'Esposito, 1999a, 1999b). Furthermore, they did not reflect a motor preparation signal, because the coordinates of the motor response vector could not be predicted during the delay epoch of one of these tasks (Postle \& D'Esposito, 1999b). Rather, we speculated that caudate nucleus activity in these studies reflected differential levels of recruitment of the motor system to support working memory performance. According to this view, one way that the nervous system stores spatial information for short periods of time is to calculate the motor response that would be required to acquire the target stimulus (e.g., with a reach or with an eye movement) and to maintain this prospective motor code during the delay epoch of the task. The position of the probe stimulus that is presented at the end of the trial can then be evaluated by comparing this stored motor code with the motor vector actually required to acquire the probe stimulus. Such a prospective motorcoding mechanism could not be used reliably to solve object working memory tasks, because there is often no direct mapping between the visual features that define a stimulus (e.g., color or physiognomy) and the motor system. This putative mechanism is consistent with psychobiological proposals of a role for the caudate nucleus in the process of egocentric localization, the mnemonic processing of egocentric spatial sensory information for its integration into a motor plan for the behavioral response (Kesner, Bolland, \& Dakis, 1993; Potegal, 1982). From the perspective of cognitive psychology, the prospective motor-coding model could potentially make an important contribution by accounting for the considerable evidence from experimental psychology that performing a secondary motor task can selectively disrupt spatial working memory performance (Baddeley, 1986; Baddeley \& Lieberman, 1980; Farmer, Berman, \& Fletcher, 1986; Hale, Myerson, Rhee, Weiss, \& Abrams, 1996; Lawrence, Myerson, Oonk, \& Abrams, 2001; Logie \& Marchetti, 1991; Quinn \& Ralston, 1986; Salway \& Logie, 1995; Smyth, Pearson, \& Pendleton, 1988). ${ }^{1}$

The present experiment tested the hypothesis that the caudate nucleus is preferentially recruited by spatial working memory tasks employing stimuli that are amenable to prospective recruitment of the motor system. We tested this hypothesis with an event-related f MRI experiment of egocentric and allocentric spatial delayed recognition. We predicted that delay-epoch activity in the caudate nucleus would be greater for egocentrically encodable spatial stimuli than for spatial stimuli that cannot be encoded egocentrically, because spatial stimuli processed in other than body-centered coordinates should be no more amenable to the creation of a prospective motor code than would be stimuli represented nonspatially. An alternative view is that the caudate nucleus, like cortical stations of the dorsal stream, might simply be preferentially recruited by any task requiring visuospatial processing. The alternative hypothesis following from this view, therefore, would predict that the caudate nucleus would be recruited to a com- parable extent by both tasks, because both are tests of spatial working memory. On egocentric (body-centered) trials, the participants judged whether the position of a single stimulus, which could be defined solely with respect to the participant's body, was identical in its target and probe presentations (Figure 1A). On the procedurally equivalent allocentric (environment-centered) trials, the participants made a comparable judgment about the horizontal distance between two stimuli (i.e., their relative spatial position; Figure 1B).

\section{METHOD}

\section{Participants}

Seven healthy adult participants ( 3 males; mean age $=25$ years) each gave informed consent prior to participation.

\section{Behavioral Task}

The two behavioral conditions were designed so that their perceptual and procedural components would be very similar and so that they would differ markedly from each other only in terms of the spatial reference frame to which they required the participants to attend. On egocentric trials, the participants first encoded the position of the target stimulus (a unitary stimulus created by joining a disk [diameter of $0.6^{\circ}$ of visual angle] to the midpoint of a vertically oriented bar $\left[2.8^{\circ}\right.$ of visual angle $\times 0.3^{\circ}$ of visual angle]), that was presented for $1 \mathrm{sec}$. After the 7-sec delay epoch, the probe stimulus (same identity as the target) appeared for $1 \mathrm{sec}$, and the participants judged whether its position matched that of the target (yes, right thumbpress; no, left thumbpress). Matches occurred with $p=.5$ (Figure 1A). The stimulus presentation procedure (detailed in the next section) ensured that the only stable referent against which a participant could assess the position of the probe, relative to the target, was his or her own body. On allocentric trials, the participants first encoded the horizontal distance between the target stimuli (disk and bar), which were presented simultaneously for $1 \mathrm{sec}$. After the 7-sec delay epoch, they judged (yes or no thumbpress) whether the horizontal distance between the probe stimuli matched the target distance. The probe stimuli always appeared at a different location on the screen (to discourage egocentric encoding) and in a different vertical position relative to each other (to discourage encoding of the two stimuli as a single object) from the target stimulus; their relative horizontal distance matched the target distance with $p=.5$ (Figure 1B). Training included explicit instructions that the nature of the target (unitary or two discrete stimuli) identified the trial type. The ranges of magnitude of offset between the probe and the target on mismatch trials (egocentric, $0.3^{\circ}-4.2^{\circ}$ of visual angle; allocentric, $0.1^{\circ}-1.4^{\circ}$ of visual angle) were determined by pilot testing, to produce comparable accuracy between the two trial types.

\section{Stimulus Presentation}

The stimuli were generated on a computer and were backprojected onto a vertical screen that was positioned approximately $310 \mathrm{~cm}$ from the participants' eyes. The participants viewed the reflection of the presentation screen off a mirror mounted on the head coil. The stimuli were presented within a white square that was nested in a black field that extended to the edge of the monitor. The entire screen display (including the black field) turned white during the delay epoch. On both trial types, when the black field, white square, and probe stimulus (or stimuli) reappeared at the end of the delay epoch, the white square was always in an unpredictably different position from where it had been at the beginning of the trial. Therefore, attending to the distance between a target stimulus and the edge of the white square would not be a successful strategy for egocentric trials. (During training, the participants were instructed 

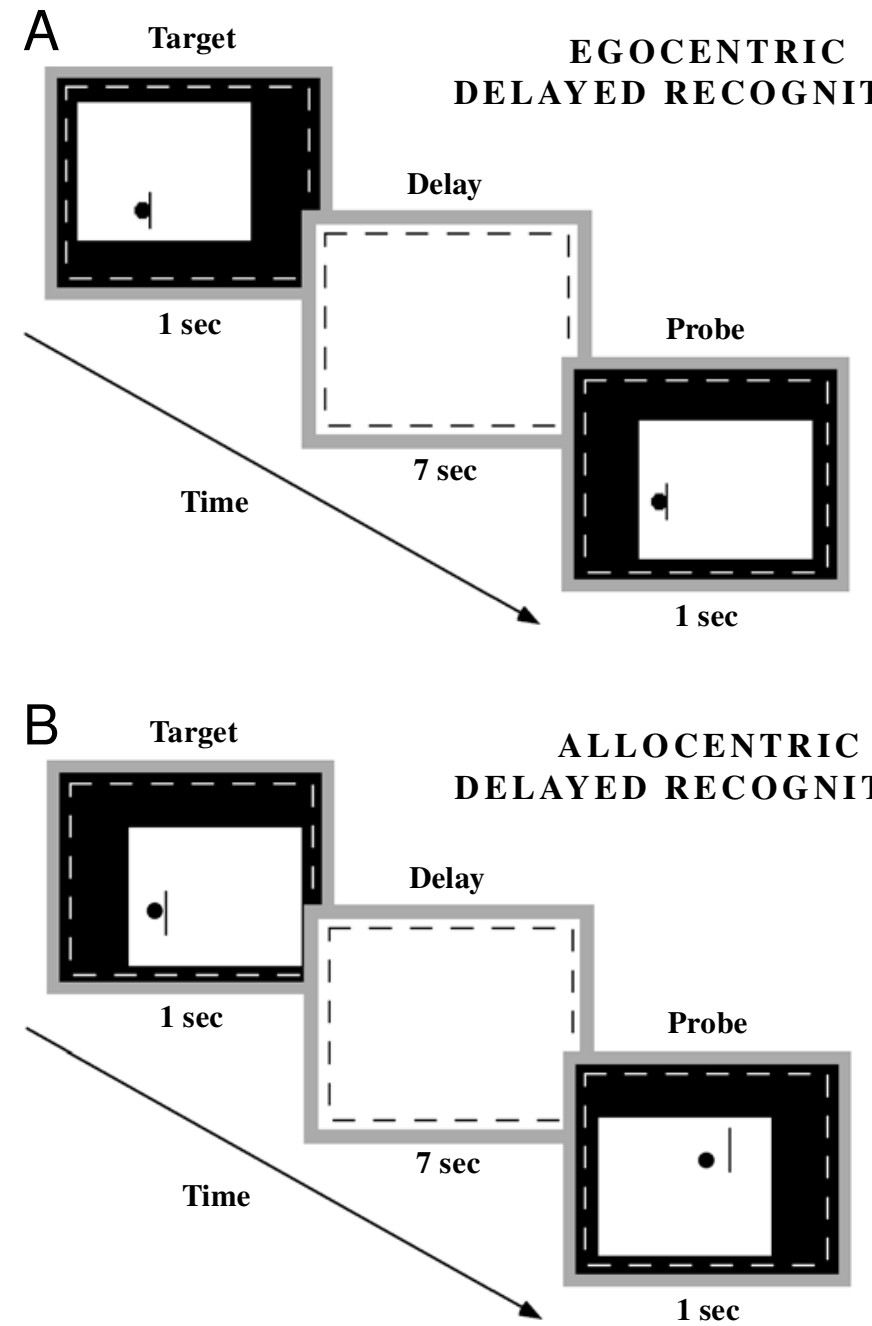

Figure 1. Schematic diagram of the visual display of the delayed-recognition tasks. Each square represents the projected image associated with an epoch of a trial: The outer gray square represents the screen beyond the projection area; the dotted line represents the boundary of the opaque filter (indiscernible to the participants during target and probe epochs); the region inside the dotted line was the only region visible to participants. In the probe epoch, the white square in which the stimuli appeared was always presented in a location that was unpredictably different from where it had appeared in the target epoch of that same trial. (A) Egocentric delayed-recognition trials required the participants to judge whether the absolute position of the probe stimulus was the same as or different from the position of the target. Because of the movement of the white square, the only reliable cue for the participant was the position of the stimulus relative to his or her body. Illustrated here is a match between the target and the probe. (B) Allocentric delayed-recognition trials required the participants to judge whether the horizontal distance between the disk and the bar was the same between target and probe epochs. Because of the movement of the white square and the fact that the center of mass of this stimulus complex always changed position unpredictably between the target and the probe, the only reliable cue was the relative horizontal distance between the disk and the bar. Illustrated here is a nonmatch between the target and the probe. (The magnitude of the difference has been exaggerated for illustrative purposes.)

to disregard this distance, because it would change between the target and the probe on every trial, regardless of trial type or trial validity.) Field of view from inside the bore of the scanner was restricted with an opaque filter attached to the opening of the bore, so that participants could see only the portion of the screen displaying the white box and the immediately surrounding black field. We confirmed this prior to each scanning session by testing whether the participant could detect the presence of an experimenter's hand when it 
was placed on the rear-projection screen at the boundary of the projected image of the computer screen. In instances in which the hand was detected, the position of the filter was changed until this portion of the visual field was occluded. This procedure was repeated for each of the four sides of the projected rectangle that corresponded to the rectangular computer screen. The phenomenological experience of the experimenters and the subjective report of several pilot participants indicated that the boundary between the opaque filter and the projected image was not detectable during epochs when the projector was projecting the black field. The effect that this created during target and probe epochs was that the white square appeared to be "floating" in an undifferentiated black field that extended to the boundaries of the participants' field of view. Thus, this procedure removed from the visual field any elements that could serve as a stable referent for the duration of a trial.

\section{Procedure}

We collected fMRI data corresponding to working memory task performance during eight scans that corresponded to eight blocks of 16 trials each. Eight egocentric and 8 allocentric delayed-recognition trials were presented per block, in a randomly determined order, yielding a total of 64 trials of each trial type per participant. Each 9sec trial was followed by an intertrial interval of $13 \mathrm{sec}$ (featureless black screen), yielding a total duration of 5 min 52 sec per block. (Total scan length was $20 \mathrm{sec}$ longer because the first trial of each block was preceded by $20 \mathrm{sec}$ of dummy gradient and radio frequency pulses in order to achieve a steady state of tissue magnetization.)

\section{fMRI Data Acquisition and Analysis}

Six participants were scanned at the Hospital of the University of Pennsylvania, and 1 was scanned at the University of WisconsinMadison. At both sites, fMRI scanning was conducted with 1.5T General Electric scanners equipped with fast gradient systems for echoplanar imaging. High-resolution sagittal and axial T1-weighted images were obtained in every participant, and gradient echo echoplanar sequences were used to acquire whole-brain data sensitive to the blood oxygen level dependent (BOLD) signal ( $\mathrm{TR}=2,000 \mathrm{msec}$, $\mathrm{TE}=50 \mathrm{msec}, \mathrm{FOV}=24 \times 24 \mathrm{~cm}$, in-plane resolution $=3.75 \times$ $3.75 \mathrm{~mm} ; 215$-mm-thick axial slices). Scans of the working memory task were preceded by a scan in which we derived an estimate of the hemodynamic response function (HRF) for each participant (Aguirre, Zarahn, \& D'Esposito, 1998). The HRF, which characterizes the fMRI response resulting from a brief impulse of neural activity (Boynton, Engel, Glover, \& Heeger, 1996), was used to convolve independent variables entered into the modified general linear model (GLM; Worsley \& Friston, 1995) that we used to analyze the results of the scans of our working memory task. Thus, each experiment proceeded in the following order: high-resolution anatomical scans; HRF derivation scan; eight working memory scans.

Our event-related design and analysis technique (Zarahn, Aguirre, \& D'Esposito, 1997) permitted assessment of variance in the fMRI signal that was attributable to the delay epoch, uncontaminated by variance arising from the target or probe epochs of the trial. (Thus, our measures of delay-epoch activity would reflect neither the mental operations of visual perception and stimulus encoding, which would be modeled by the target covariate, nor those of visual perception [of the probe], decision, response selection, and motor response, which would be modeled by the probe covariate.) The principle of this technique was to model the fMRI signal changes occurring during the three discrete epochs of the behavioral trials (target, delay, and probe) with covariates consisting of shifted BOLD HRFs (Postle, Zarahn, \& D'Esposito, 2000; Zarahn et al., 1997). Between-condition differences in $\mathrm{fMRI}$ signal were tested with contrasts of the coefficients associated with the covariates in question. Of primary theoretical interest was the comparison of delay-epoch activity in the egocentric condition with delay-epoch activity in the allocentric condition, which was implemented in our analyses with the two-tailed contrast [Delay ${ }_{\text {Egocentric }}-$ Delay $_{\text {Allocentric }}$ ], from which a result with a positive value would indicate greater delay-epoch activity in egocentric than in allocentric trials and a result with a negative value would indicate the converse. In the event that these analyses confirmed our hypothesis, we planned to test the temporal specificity of this result by also testing for differential levels of targetand probe-specific activity. Our analyses proceeded in three steps: single-participant analyses, functionally guided group analyses, and structurally guided group analyses.

\section{Regions of Interest}

All our analyses were performed with reference to regions of interest (ROIs). All structural ROIs described in this report were drawn by the first author, always before he had seen statistical maps from the participant in question. Caudate nucleus and putamen ROIs were drawn for each participant on that participant's T1 anatomical images. Caudate nucleus ROIs incorporated the head (beginning rostrally and ventrally at approximately the level of the anterior commissure) and the body (extending caudally along the lateral wall of the lateral ventricle and ending at the ventral-most level at which the body of the lateral ventricle appeared intact in one slice) of this structure. Analyses of cortical ROIs were not required to assess the principal hypothesis of interest in this study, because no cortical region had displayed activity consistent with a prospective motorcoding function in our previous studies (Postle \& D'Esposito, 1999a, 1999b). The effect of our experimental manipulation on delay-epoch activity in regions of the cortex implicated in spatial processing is, nonetheless, of broader interest, and so these analyses were included in our report. The cortical ROIs were selected because these regions had been identified by previous studies of spatial frames of reference (e.g., Andersen, Essick, \& Siegel, 1985; Butters, Soeldner, \& Fedio, 1972; Galati et al., 1999; Graziano, Yap, \& Gross, 1994; Pohl, 1973; Rafal \& Robertson, 1995; Semmes, Weinstein, Ghent, \& Teuber, 1963; Snyder, Grieve, Brotchie, \& Andersen, 1998; Vallar et al., 1999). They were the dorsolateral prefrontal cortex (DLPFC; corresponding to Brodmann areas (BAs) 9 and 46, incorporating the middle frontal gyrus and a portion of the inferior frontal gyrus); the ventrolateral prefrontal cortex (VLPFC; corresponding to BAs 44, 45, and 47 , incorporating the portion of the inferior frontal gyrus ventral to area 46 and anterior to the precentral sulcus); the superior frontal cortex (SFC; corresponding to BA 8, incorporating the superior frontal gyrus and the superior frontal sulcus, bounded caudally by voxels located $\geq 6 \mathrm{~mm}$ rostral to the fundus of the precentral sulcus [PCS]); the medial premotor cortex (mPMC; defined as the cortex of BA 6 that lay within the midline or was contiguous with this midline cortex, bounded ventrally by the cingulate sulcus); the lateral PMC (IPMC; corresponding to lateral BA 6, defined as the cortex in the rostral bank of the PCS, bounded rostrally in its ventral aspect by the prefrontal cortex and in its dorsal aspect by the SFC and medially by the superior frontal gyrus); the portion of the superior parietal lobule (SPL) corresponding to BA 7 (bounded rostrally by the postcentral sulcus and laterally by the fundus of the intraparietal sulcus [IPS]); the portion of the inferior parietal cortex (IPC) corresponding to BAs 40 and 39 (bounded medially by the fundus of the IPS); and extrastriate occipital and temporal areas corresponding to BAs 19,18 , and 37 (bounded dorsally by the inferior temporal sulcus and rostrally by the lateral temporooccipital sulcus and the imaginary continuation of this landmark to the lingual or anterior calcarine sulcus, depending on the dorsoventral position of a particular slice). We created these ROIs by drawing them onto the "canonical" representation of a brain in Talairach space that is provided in SPM96b, using the atlas of Talairach and Tournoux (1988) to determine the boundaries between BAs. Next, we transformed these ROIs from Talairach space into the native space in which each participant's data had been acquired, by applying an algorithm for 12-parameter affine transformation (Friston et al., 1995) with non- 
linear deformations (Ashburner \& Friston, 1996). Finally, because this method of reverse normalizing ROIs from standard to native space rarely yields a seamless volume and because there is known to be considerable subject-to-subject variability in the precise location of BAs (e.g., Rajkowska \& Goldman-Rakic, 1995), we adjusted each ROI to conform precisely with each participant's anatomy, employing several atlases (Damasio, 1995; Duvernoi, 1999; Mai, Assheuer, \& Paxinos, 1997).

\section{Single-Participant Analyses}

Single-participant analyses maintain the high spatial resolution afforded by $\mathrm{fMRI}$ and permit detection of interparticipant variability (Postle, Zarahn, \& D'Esposito, 2000). Previous studies of spatial working memory that, like the present study, featured a large number of observations per participant (and therefore, a large number of degrees of freedom in contrasts performed on single-participant data) have demonstrated ample sensitivity to detect signal intensity changes of interest, particularly in the cortex (Postle, Berger, Taich, \& D'Esposito, 2000; Postle \& D'Esposito, 1999a, 1999b, 2000; Zarahn et al., 1997, 1999). Single-participant analyses in the present study featured in excess of 1,200 effective degrees of freedom. In these analyses, we performed the two-tailed contrast [Delay ${ }_{\text {Egocentric }}$ - Delay Allocentric $]$ in the caudate nucleus and the putamen, the regions of principal interest to our hypothesis.

The results of previous studies of caudate nucleus activity in working memory tasks (Postle \& D'Esposito, 1999a, 1999b) suggest that the single-participant approach may lack sensitivity in the subcortex. We have found that effects in the caudate nucleus were sufficiently subtle that they did not manifest themselves as suprathreshold activity in each individual participant, although reliable patterns of effects across participants (suprathreshold for some, subthreshold for others) emerged in the group analyses. For the present study, we were particularly cognizant of the issue of sensitivity, not only because our principal ROI was in a subcortical region in which differences in signal might be smaller than those in the cortex, but also because the two conditions that we were contrasting - both spatial delayed recognition - were so similar. (Our previous studies of basal ganglia have contrasted delay-evoked responses between spatial [Postle \& D'Esposito, 1999b] or spatiomotor [Postle \& D'Esposito, 1999a] tasks and nonspatial tasks.) Therefore, we expected that our group analyses were more likely to represent the decisive tests of our hypothesis.

\section{Group Analyses}

We performed two types of group analyses: functionally guided and structurally guided. These random effects group analyses were performed with individual data sets that were retained in the native space in which the data were collected. That is, we neither normalized nor averaged the data. The normalization-then-averaging approach to group analyses requires spatial smoothing of the data and is constrained to identifying as significant the foci of activation that overlap topographica lly across participants (Brett, Johnsrude, \& Owen, 2002). Neither this requirement nor this constraint seemed appropriate for analyses focused on the caudate nucleus, a structure for which smoothing risks incorporating spurious signal changes attributable to the brain-ventricle interface, and on the putamen, and in which previous analyses have revealed topographically heterogeneous patterns of activity across participants (Postle \& D'Esposito, 1999a).

Functionally guided group analyses. These were performed by identifying functionally the voxels within each ROI, from each participant's data, that were active during a specific epoch of the delayedrecognition task, extracting an index of the experimental effect from these voxels, and then assessing the reliability of these indices across participants. The analyses proceeded in four steps: first, identifying within each participant the voxels that demonstrated a significant main effect of activity (when collapsed across trial type) for the trial epoch in question with the contrast $\left[\mathrm{Epoch}_{\text {Egocentric }}+\mathrm{Epoch}_{\text {Allocentric }}\right]$; second, extracting from these voxels an average time series for each participant; third, assessing the relative magnitude of egocentric versus allocentric activity by applying to this time series the orthogonal two-tailed contrast [Epoch Egocentric - Epoch $_{\text {Allocentric }}$ ]; and fourth, entering the resultant $t$ values from this contrast (one for each participant) into a paired $t$ test. If, for a particular participant, no voxels were identified with the $\left[\right.$ Epoch $_{\text {Egocentric }}+$ Epoch $\left._{\text {Allocentric }}\right]$ contrast in Step 1, this participant's data were excluded from this analysis (although not from the study, as is detailed in the next section). (A rationale for employing $t$ values as indices of fMRI effects and as dependent values in group analyses is provided elsewhere [Postle, Zarahn, \& D'Esposito, 2000].)

Structurally guided group analyses. These analyses differed from the functionally guided analyses in that they collapsed across all voxels within each ROI. Thus, they were not restricted to voxels that were active during the epoch in question. The structurally guided group analyses were planned for three reasons. First, they would serve as confirmatory group analyses in that they would include the data from all the participants, in the event that data were dropped from the functionally guided group analysis. Second, they would serve a distinct confirmatory purpose, in that they were not subject to a possible source of bias that existed in the functionally guided group analysis - the selection bias of testing only critical voxels that were identified with an omnibus test of activity during a particular epoch. Third, in the event that our hypothesis was confirmed by the functionally guided group analysis, they would test the relative importance of egocentric versus allocentric delay-epoch activity across the entire extent of the head and body of the caudate nucleus. The latter reason was motivated in part by evidence from the monkey caudate nucleus that working-memory-related function for different stimulus domains may be supported in a topographically segregated manner (Levy, Friedman, Davachi, \& Goldman-Rakic, 1997). Whole-ROI analyses were physiologically naive because, by pooling signals from every voxel in an ROI, they undoubtedly reflected contributions from many voxels whose function was unrelated to our working memory task. We reasoned, however, that this analysis would be sensitive to allocentric delay-epoch activity if this activity were as important a feature of caudate nucleus function as is egocentric delayepoch activity but more diffuse and, thus, detectable only by an analysis sensitive to activity across the entire structure. Were this the case, results of two-tailed contrasts of [Delay ${ }_{\text {Egocentric }}-$ Delay $_{\text {Allocentric }}$ ] on the whole-ROI time series from each participant would be expected to be near zero, and the group mean of these effects would not be significantly different from zero. For this structurally guided group analysis, we extracted a single spatially averaged time series from the caudate nucleus ROI of each participant (by collapsing across all voxels in the ROI), interrogated each time series with the

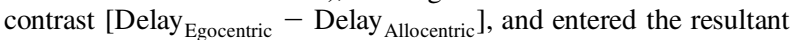
measures of relative importance of egocentric versus allocentric delay-epoch activity into a paired $t$ test.

\section{RESULTS}

\section{Behavior}

Accuracy was comparable in the two conditions (egocentric $=79.9 \%, S D=13.0 \%$; allocentric $=80.7 \%, S D=$ $10.2 \%$ ), but response time (RT) was not [egocentric $=$ $941.2 \mathrm{msec}, S D=363.5$; allocentric $=1,110.4 \mathrm{msec}$, $S D=292.0 ; t(6)=-3.2, p<.05]$.

\section{Caudate Nucleus}

Single-participant analyses. One participant in our sample demonstrated voxels in the caudate nucleus whose 
A
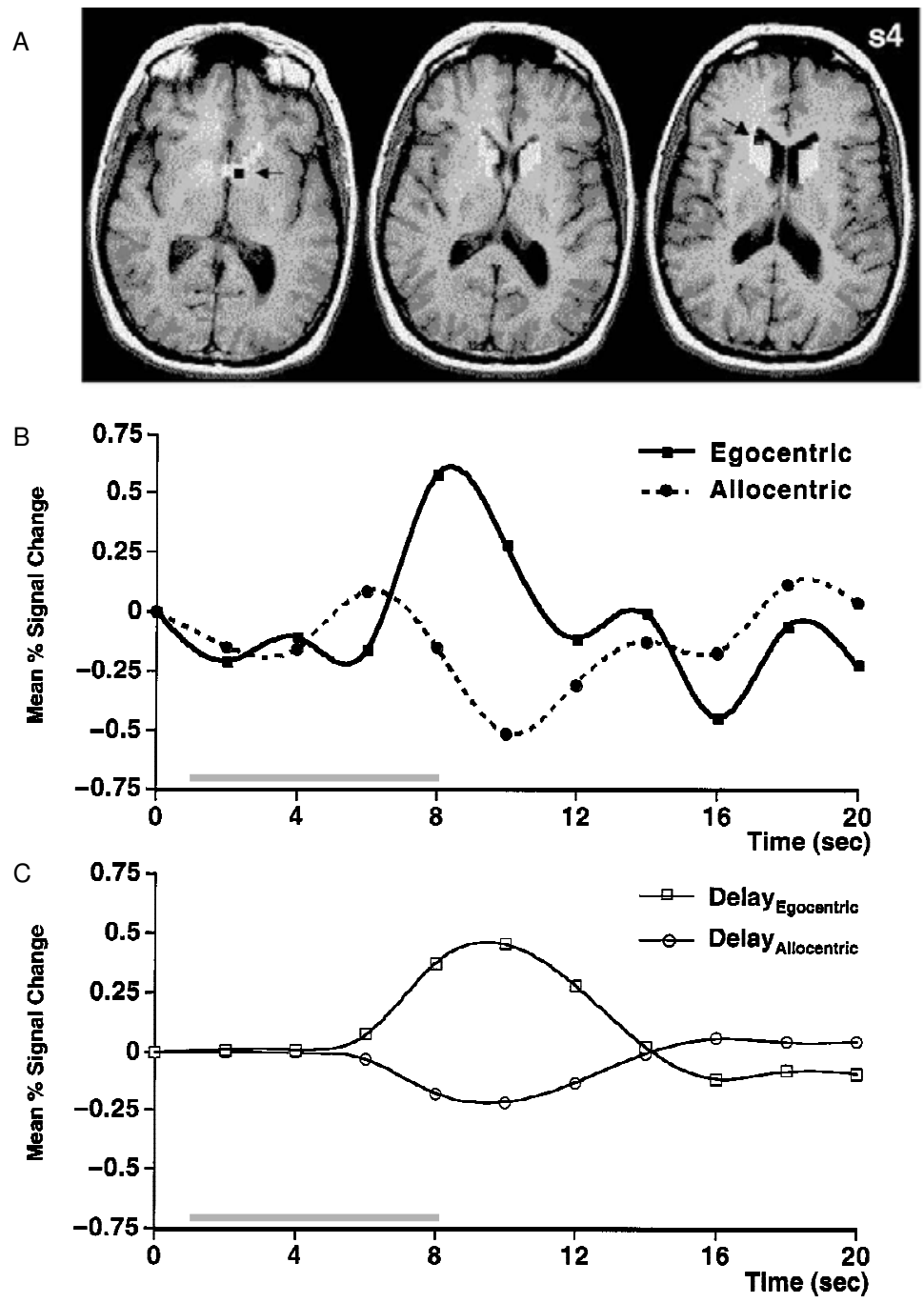

Figure 2. Significantly greater egocentric than allocentric delay-epoch activity in the caudate nucleus of Participant 4. (A) Locus of activation of the two suprathreshold voxels [black squares identified with arrows; $t(1204)=5.0, p<$ $.0001]$. The caudate nucleus region of interest (ROI) is depicted in translucent white overlaid on the structural T-1 images; "gaps" in ROI in the right hemisphere reflect areas in which the fMRI signal was too low to measure, due to susceptibility artifact. (B) fMRI time series data from these two voxels, trial averaged for each of the two trial types; the gray bar represents the duration of the delay epoch. (C) Illustration of the least-squares solution of the general linear model for the delay-epoch effects from these two voxels. Each plot represents a delay-epoch covariate scaled by its parameter estimate. These covariates model delay-epoch activity taking place at Time 4; note how they take into account the sluggishness of this participant's hemodynamic response, which peaks approximately 6 sec after onset. The slight negative weighting on the Delay $_{\text {Allocentric }}$ covariate indicates that there was no detectable delay-epoch activity in these voxels on allocentric trials. The gray bar represents the duration of the delay epoch.

delay-epoch activity was significantly greater in the egocentric than in the allocentric condition (Figure 2). (That this effect exceeded threshold only in the data of 1 participant was independent of whether it might also be present at subthreshold levels in the majority of the participants. The group analyses that follow represent tests for the reli- ability of such subthreshold effects across our sample of participants.) No participants' data showed the opposite pattern.

Functionally guided group analysis. Voxels demonstrating a significant main effect of delay-epoch activity, identified with the contrast [Delay Egocentric + Delay $_{\text {Allocen- }}$ tric], were identified in 5 participants (Figure 3A). Assess- 
A

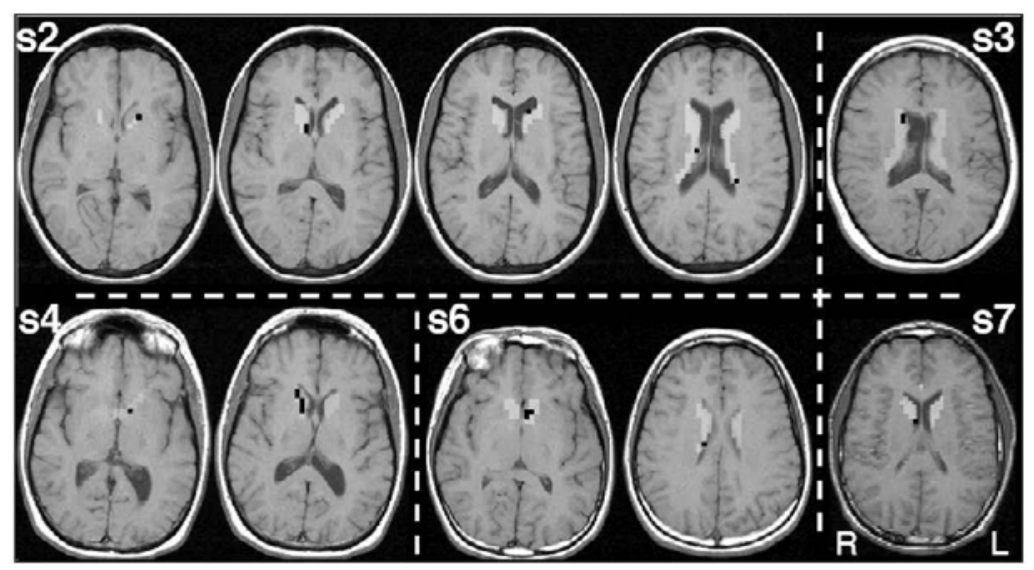

B

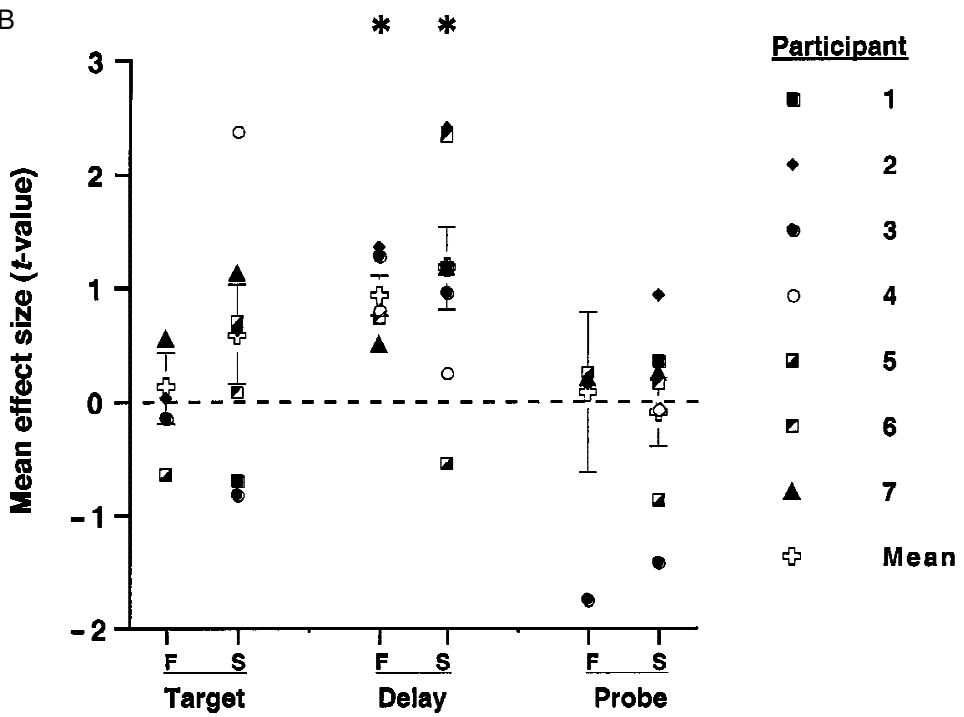

Figure 3. Caudate nucleus data from the group analyses. (A) Caudate nucleus voxels (depicted in black) identified by the functionally guided group analysis in 5 participants. These voxels demonstrated a significant main effect of delay-epoch activity (defined with the contrast [Delay ${ }_{\text {Egocentric }}+$ Delay $\left._{\text {Allocentric }}\right]$ ). The regions of interest appear as translucent white overlays superimposed on the structural images; data from different participants are separated by dashed lines. (B) Results of the two-tailed [Epoch $_{\text {Egocentric }}-$ Epoch $_{\text {Allocentric }}$ contrasts, illustrated for individualparticipants and the group means, reveal that the spatial reference frame manipulation affected caudate nucleus activity reliably only during the delay epoch of the task. Positive values indicate that egocentric activity $>$ allocentric activity; negative values indicate the converse. $F$, functionally guided group analy sis; $S$, structurally guided group analysis. Error bars represent $S E M$. Asterisks (*) indicate statistically significant effects.

ment of activity in these voxels with the orthogonal contrast $\left[\right.$ Delay ${ }_{\text {Egocentric }}-$ Delay $\left._{\text {Allocentric }}\right]$ indicated that the egocentric delay-epoch effect was greater than the allocentric delay-epoch effect in each of these 5 participants, a significant effect $[t(4)=5.6, p<.01$; Figure $3 \mathrm{~B}]$. (Although the egocentric delay-epoch activity was greater, there was reliable allocentric delay-epoch activity in these voxels in each of these 5 participants.) Additional functionally guided group analyses of caudate nucleus activity during nondelay portions of the trial failed to find evidence for an effect of reference frame during either the target epoch or the probe epoch of the trial (Figure 3B).

Structurally guided group analysis. This analysis indicated that delay-epoch activity across the entire caudate nucleus ROI was greater on egocentric than on allocentric trials in 6 of 7 participants, also a significant effect $[t(6)=$ $3.3 ; p<.05$; Figure 3B]. As with the functionally guided group analyses, additional structurally guided group analy- 
Table 1

Results of Functionally Guided Analyses in Cortical Regions of Interest (ROIs; Mean Effect of [Delay Egocentric $_{\text {illocentric }}$ - Delay Contrasts)

\begin{tabular}{lrrr}
\hline \multirow{2}{*}{ ROI } & \multicolumn{2}{c}{$t$ value } & \\
\cline { 2 - 3 } & $M$ & $S E M$ & $N$ \\
\hline DLPFC & 1.91 & .77 & 7 \\
VLPFC & 1.26 & .67 & 6 \\
SFC & .04 & .75 & 6 \\
mPMC & 1.45 & 1.04 & 6 \\
IPMC & 1.78 & $.82^{*}$ & 6 \\
SPL & 2.11 & 1.12 & 7 \\
IPC & 2.30 & 1.46 & 6 \\
Extrastriate & 1.83 & 1.20 & 7 \\
\hline
\end{tabular}

Note $-N=$ number of participants for whom delay-epoch activity was detected. DLPFC, dorsolateral prefrontal cortex; VLPFC, ventrolateral prefrontal cortex; SFC, superior frontal cortex; mPMC, medial premotor cortex; IPMC, lateral premotor cortex; SPL, superior parietal lobule; IPC, inferior parietal cortex. ${ }^{*} p=.08$.

ses also failed to reveal evidence for an effect of reference frame during either the target epoch or the probe epoch of the trial (Figure 3B).

\section{Putamen}

Single-participant analyses. These yielded null results in all the participants.

Functionally guided group analysis. Four of 7 participants demonstrated a significant main effect of delayepoch activity in this ROI: Egocentric delay-epoch activity was greater in 2 of these participants; allocentric delayepoch activity was greater in the other 2 .

Structurally guided group analysis. The ROI-wide group analysis revealed reliably greater egocentric than allocentric delay-epoch activity in the putamen $[t(6)=2.8$, $p<.05]$.

\section{Cortex}

Functionally guided group analyses. We also performed functionally guided group analyses in several cortical ROIs (identified in the Method section) that were selected to incorporate the regions identified by previous neuropsychological(Butters et al., 1972; Pohl, 1973; Rafal \& Robertson, 1995; Semmes et al., 1963) and neurophysiological (Andersen et al., 1985; Galati et al., 1999; Graziano et al., 1994; Snyder et al., 1998; Vallar et al., 1999) studies of egocentric and allocentric spatial behavior. The only cortical ROI in which delay-epoch effects approached significance was a trend toward greater egocentric than allocentric delay-epoch activity in the IPMC (Table 1, Figure 4). (It bears reiterating here that, in all of these regions, there was significant delay-epoch activity, as identified by the contrast [Delay Egocentric + Delay $_{\text {Allocentric }}$. These null results are best interpreted as indicating that this activity was not significantly greater in one condition than in the other.) Because of the trend in the IPMC and because we did not find significant effects in the caudate nucleus in the probe epoch of the trial, we also examined probe-related effects in voxels identified with the contrast Probe $_{\text {Egocen- }}$ tric + Probe $\left._{\text {Allocentric }}\right]$ in this region: Probe-related activity in the IPMC was significantly greater in egocentric than in allocentric trials $[t(4)=5.7 ; p<.005$; Figure 4]. (No PMC voxels were identified in Participant 6 with either the $\left[\right.$ Delay $_{\text {Egocentric }}+$ Delay $\left._{\text {Allocentric }}\right]$ or the $\left[\right.$ Probe $_{\text {Egocentric }}+$ Probe $\left._{\text {Allocentric }}\right]$ contrast. Due to a technical problem, probe-related activity in the PMC could not be determined in Participant 5. Similar to the case with caudate nucleus delay-epoch activity, there was reliable allocentric probeepoch activity in the voxels identified for this functionally guided analysis in each of these 5 participants.)

\section{DISCUSSION}

Our results revealed greater caudate nucleus delay-epoch activity in egocentric than in allocentric trials of a spatial working memory task, as assessed by two types of group analyses. These results cannot be interpreted as an effect of difficulty, because the RT data suggest that the allocentric task may have been the more difficult of the two. These results confirmed our hypothesis, indicating that this structure is differentially engaged by working memory for different types of spatial stimuli. This effect was limited to the delay epoch of the delayed-recognition trials and, thus, was likely independent of encoding- and response-related processes. The results in the putamen were equivocal: The functionally guided group analysis yielded a null result, but the structurally guided group analysis yielded a positive result. Thus, developing a clear understanding of the role of the putamen in spatial working memory requires further study. In the $1 \mathrm{PMC}$ - an ROI that incorporated the frontal eye fields, as well as arm and hand representations - the predominance of activity on egocentric trials approached significance in the delay epoch and achieved significance in the probe epoch. This represents the strict limit of what we can conclude from our results. What follows is a consideration of the implications of these results for the theoretical considerations that motivated this study.

Taken together, these results are consistent with the view that the caudate nucleus mediates participation of the motor system in spatial working memory performance when stimuli can be encoded in a body-centered reference frame. A precedent for this prospectivemotor-coding model comes from evidence that damage to the caudate nucleus in rats disrupts egocentric localization, the ability to integrate working memory of egocentric (but not allocentric) spatial information into a motor plan (Kesner et al., 1993).

A previous behavioral study in humans has generated evidence for the independence of working memory performance with body-versus environment-centered spatial information: Pointing to a set of distractors defined with respect to the body selectively disrupted egocentric delayedresponse performance, whereas pointing to a set of environmentally defined distractors selectively disrupted allocentric delayed-response performance (Woodin \& Allport, 1999). The present results suggest a neuroanatomical basis for this behavioral dissociation. They are also con- 
A

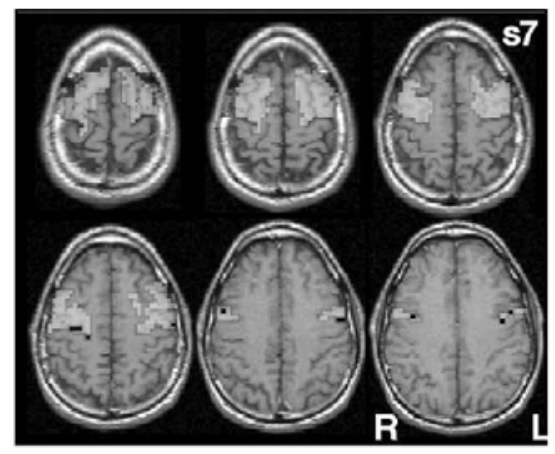

B

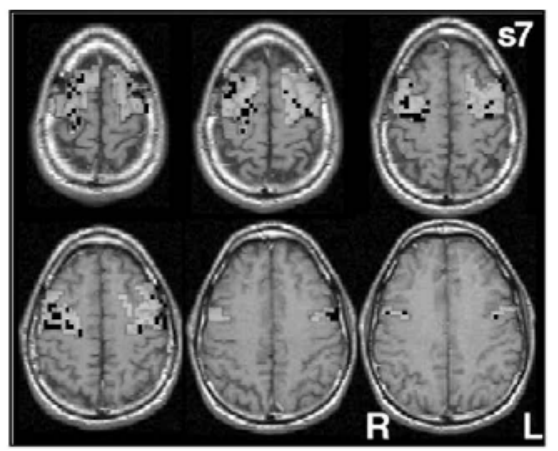

*

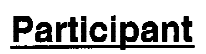

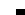

$+2$

- 3

$\circ 4$

5

$1 \quad 7$

↔ Mean

Figure 4. Premotor cortex (PMC) data from the functionally guided group analyses. (A) Lateral PMC voxels (black squares) in Participant 7 , demonstrating a significant main effect of delay-epoch activity. The region of interest (ROI) is depicted in translucent white overlay. (B) Lateral PMC voxels (black squares) in Participant 7, demonstrating a significant main effect of probe-epoch activity. The ROI is depicted in translucent white overlay. (C) Results of the two-tailed [Epoch Egocentric - Epoch $_{\text {Allocentric }}$ contrasts, illustrated for individual participants and the group means, reveal that the spatial reference frame manipulation affected lateral PMC activity to a marginally significant extent during the delay epoch $(p=$ $.08)$ and to a significant extent during the probe epoch $(p<.01)$. Positive values indicate that egocentric activity $>$ allocentric activity; negative values indicate the converse. Error bars represent $S E M$, although for the probe epoch data they are too small to be visible. An asterisk (*) indicates a statistically significant effect.

sistent with the idea that a prospective motor-coding mechanism may contribute to spatial working memory in situations in which the stimuli are encodable egocentrically. The interruption of such a mechanism might explain the selective disruptive effect of secondary motor activity on spatial working memory performance (concurrent finger tapping [Farmer et al., 1986; Salway \& Logie, 1995; Smyth et al., 1988], pointing [Hale et al., 1996], eye movements [Baddeley, 1986; Hale et al., 1996; Lawrence et al., 2001], or arm movements [Baddeley \& Lieberman, 1980; Lawrence et al., 2001; Logie \& Marchetti, 1991; Quinn \&
Ralston, 1986]). Previously, Baddeley and Logie (1999) have interpreted these motor disruption data in the context of the distinction between visual working memory for what versus where aspects of a visual scene. In their view, the visuospatial scratch pad of working memory (Baddeley \& Hitch, 1974; Logie, 1995) is subdivided into a visual cache, responsible for retaining visual patterns, and an inner scribe, responsible for retaining spatial information, including sequences of "movement or of paths between objects and locations" (Baddeley \& Logie, 1999, p. 36). One might be tempted, therefore, to interpret the present 
results as suggestive of neuroanatomical and neurophysiological bases for the inner scribe. But our results cannot be completely accounted for by the model of the inner scribe as it is currently understood, because they (along with those of Woodin \& Allport, 1999) indicate at least partial independence of processes supporting working memory for spatial information that is represented in different reference frames. The inner scribe, in contrast, is described as being responsible for all types of spatial and motoric working memory function (Baddeley \& Logie, 1999).

On the basis of our results, we suggest that short-term retention of spatial information can be accomplished by at least two distinct mechanisms: one featuring the maintenance of a sensory code (e.g., Awh, Jonides, \& ReuterLorenz, 1998; Logie, 1995), the second featuring the transformation of sensory information into a motor code and the maintenance of this prospective motor code during the delay epoch. The sensory coding mechanism is supported in part by attention-based rehearsal-the allocation of spatial selective attention to visually topographically organized regions of the extrastriate cortex (Awh, AnlloVento, \& Hillyard, 2000; Awh \& Jonides, 2001; Awh et al., 1999). In addition to these regions, several parietal, frontal, and subcortical areas have also been identified in a network that is activated during spatial working memory tasks (the SPL and the inferior parietal lobule, the DLPFC, the SFC, the frontal eye fields and other areas of the IPMC, and the caudate nucleus; see, e.g., Corbetta, Kincade, \& Shulman, 2002; Leung, Gore, \& Goldman-Rakic, 2002; Postle, Berger, et al., 2000; Postle \& D'Esposito, $1999 \mathrm{~b})$. Some of the activity in these regions is also likely to reflect the operation of a sensory-coding mechanism. For example, the caudate nucleus and the IPMC were active during the allocentric trials of our experiment, and we believe that retention of a sensory code is the way that the participants performed this task. In contrast to the sensory code, our results suggest that the prospective motor code may rely on a sensory-motor transformation that is mediated by the caudate nucleus and, possibly, by virtue of reciprocal connections with the caudate nucleus, regions of the PMC. Maintenance of this motor code during the delay epoch might also rely on neostriatal-PMC interactions.

It is important to note that the egocentric task could, in principle, be solved with a purely sensory-coding mechanism. By this interpretation, the neurophysiological differences that we observed in our study might be due solely to differences between the computational demands of an egocentric spatial sensory code and those of an allocentric spatial sensory code. (Note, however, that the accuracy and RT data argue against a simple difference in difficulty, because the latter suggest that the allocentric task may have been more difficult than the egocentric task.) Consistent with the idea that both tasks recruited a sensorycoding mechanism is that fact that in all of the cortical ROIs that we interrogated, except the IPMC, we did not find any differences between delay-period activity on egocentric versus allocentric trials. But recruitment of a prospective motor-coding mechanism or of a sensory-coding mech- anism need not be mutually exclusive processes. It may be that a prospective motor-coding mechanism is recruited in addition to a sensory-coding mechanism when the stimulus configuration permits it, as did the egocentric trials in our study. By this interpretation, the greater signal that we observed in the caudate nucleus and in the IPMC on egocentric trials may have resulted from the additive effects of two mechanisms supporting performance on these trials, as contrasted with only one mechanism supporting performance on allocentric trials. It is certainly plausible, even likely, that any given brain structure can support multiple distinct cognitive mechanisms (D'Esposito, Ballard, Aguirre, \& Zarahn, 1998). Thus, it is reasonable to assume that the caudate nucleus and the IPMC could support both the sensory-coding and the prospective motor-coding mechanisms of spatial working memory. The relatively coarse spatial resolution of our data precludes speculation about whether or not the same neural circuits within these regions might contribute to both functions. Bearing in mind this inferential limitation, it is nonetheless worth noting that our results are inconsistent with a segregation-of-function model, in which one region of the caudate nucleus is relatively specialized for egocentric working memory functions and another for allocentric working memory functions. This is because our structurally guided group analysis, which collapsed across the entire caudate nucleus ROI, revealed reliably greater egocentric than allocentric delay-epoch activity. Conclusive resolution of such questions about the fine-grained topography of prospective motor-coding versus egocentric sensory-coding versus allocentric sensorycoding functions of the caudate nucleus, however, must await investigation with methods featuring higher spatial resolution than that afforded by fMRI at 1.5 tesla.

The results from our $\mathrm{ARI}$ investigation of what-thenwhere delayed-recognition performance (Postle \& D'Esposito, 1999a, 1999b) suggest that the prospective motorcoding mechanism may be of particular utility when a motor response will be contingent upon a spatial memory. Concrete examples of such a motor code might be the computation and maintenance of the eye movement vector required to foveate the target stimulus or of the arm or hand movement vector required to reach or point to it (Cheffi, Allport, \& Woodin, 1999). A prospective motor-coding mechanism need not contribute directly to response execution. For example, the responses required by our tasks did not map directly to the configuration of the stimuli. That is, neither the motor coordinates required to acquire a target with a saccade nor those required for a grasp mapped directly to the yes/no buttonpress required of the participants. The spatial information stored in a motor code, therefore, might be expected to contribute to the decision-making processes prompted by the onset of the probe stimulus (e.g., by assessing the error signal between the remembered targetrelated motor code and the probe-acquiring motor code), rather than directly to the response execution processes triggered by the outcome of this decision. Our results suggest that this decision-making process depends primarily on the frontal cortex, rather than on the neostriatum. 


\section{REFERENCES}

Aguirre, G. K., Zarahn, E., \& D'Esposito, M. (1998). The variability of human, BOLD hemodynamic responses. NeuroImage, 8, 360-369.

Andersen, R. A., Essick, G. K., \& Siegel, R. M. (1985). Encoding of spatial location by posterior parietal neurons. Science, 230, 456-458.

Ashburner, J., \& Friston, K. (1996). Fully three-dimensional nonlinear spatial normalization: A new approach. NeuroImage, 3, S111.

Awh, E., Anllo-Vento, L., \& Hillyard, S. A. (2000). The role of spatial selective attention in working memory for locations: Evidence from event-related potentials. Journal of Cognitive Neuroscience, $\mathbf{1 2}$, 840-847.

AwH, E., \& JonIDEs, J. (2001). Overlapping mechanisms of attention and spatial working memory. Trends in Cognitive Sciences, 5, 119-126.

Awh, E., Jonides, J., \& Reuter-Lorenz, P. A. (1998). Rehearsal in spatial working memory. Journal of Experimental Psychology: Human Perception \& Performance, 24, 780-790.

Awh, E., Jonides, J., Smith, E. E., Buxton, R B., Frank, L. R, Love, T., Wong, E. C., \& GMeIndL, L. (1999). Rehearsal in spatial working memory: Evidence from neuroimaging. Psychological Science, 10, 433-437.

BADDELEY, A. D. (1986). Working memory. London: Oxford University Press.

BADDEley, A. D., \& Hitch, G. J. (1974). Working memory. In G. H. Bower (Ed.), The psychology of learning and motivation (Vol. 8, pp. 47-89). New York: Academic Press.

BAdDEley, A. D., \& Lieberman, K. (1980). Spatial working memory. In R. S. Nickerson (Ed.), Attention and performance VIII (pp. 521539). Hillsdale, NJ: Erlbaum.

BADDELEy, A. D., \& LogIE, R. H. (1999). Working memory: The multiplecomponent model. In A. Miyake \& P. Shah (Eds.), Models of working memory (pp. 28-61). Cambridge: Cambridge University Press.

Battig, K., Rosvold, H. E., \& Mishrin, M. (1960). Comparison of the effects of frontal and caudate lesions on delayed response and alternation in monkeys. Journal of Comparative \& Physiological Psychology, 53, 400-404.

Boynton, G. M., Engel, S. A., Glover, G. H., \& Heeger, D. J. (1996). Linear systems analysis of functional magnetic resonance imaging in human V1. Journal of Neuroscience, 16, 4207-4221.

Brett, M., Johnsrude, I. S., \& Owen, A. M. (2002). The problem of functional localization in the human brain. Nature Reviews: Neuroscience, 3, 243-249.

Butters, N., Soeldner, C., \& Fedio, P. (1972). Comparison of parietal and frontal lobe spatial deficits in man: Extrapersonal vs. personal (egocentric) space. Perceptual \& Motor Skills, 34, 27-34.

Cheffi, S., Allport, D. A., \& Woodin, M. (1999). Hand-centered coding of target location in visuo-spatial working memory. Neuropsychologia, 37, 495-502.

Corbetta, M., Kincade, J. M., \& Shulman, G. L. (2002). Neural systems for visual orienting and their relationships to spatial working memory. Journal of Cognitive Neuroscience, 14, 508-523.

Courtney, S. M., Ungerleider, L. G., Keil, K., \& HaXby, J. (1996). Object and spatial visual working memory activate separate neural systems in human cortex. Cerebral Cortex, 6, 39-49.

Damasio, H. (1995). Human brain anatomy in computerized images. Oxford: Oxford University Press.

DEAN, W. H., \& DAVIS, G. D. (1959). Behavior changes following caudate lesions in rhesus monkey. Journal of Neurophysiology, 22, 525-537.

Della Sala, S., Gray, C., Baddeley, A., Allamano, N., \& Wilson, L. (1999). Pattern span: A tool for unwelding visuo-spatial memory. Neuropsychologia, 37, 1189-1199.

D’Esposito, M., Aguirre, G. K., Zarahn, E., \& Ballard, D. (1998). Functional MRI studies of spatial and non-spatial working memory. Cognitive Brain Research, 7, 1-13.

D’Esposito, M., Ballard, D., Aguirre, G. K., \& Zarahn, E. (1998). Human prefrontal cortex is not specific for working memory: A functional MRI study. NeuroImage, 8, 274-282.

Divac, I., Rosvold, H. E., \& Szwarcbart, M. K. (1967). Behavioral effects of selective ablation of the caudate nucleus. Journal of Comparative \& Physiological Psychology, 63, 184-190.

DuvernoI, H. M. (1999). The human brain: Surface, blood supply, and three-dimensional sectional anatomy (2nd ed.). New York: SpringerVerlag.

Farmer, E. W., Berman, J. V. F., \& Fletcher, Y. L. (1986). Evidence for a visuo-spatial scratch-pad in working memory. Quarterly Journal of Experimental Psychology, 38A, 675-688.

Friston, K. J., Ashburner, J., Frith, C. D., Poline, J.-B., Heather, J. D., \& Frackowiak, R. S. J. (1995). Spatial registration and normalization of images. Human Brain Mapping, 2, 165-189.

Galati, G., Lobel, E., Berthoz, A., Pizzamiglio, L., Le Bihan, D., \& VAllar, G. (1999). Egocentric and allocentric coding of space in the human brain. NeuroImage, $9, \mathrm{~S} 745$.

Goldman, P. S., \& Rosvold,H. E (1972). The effects of selective caudate lesions in infant and juvenile rhesus monkeys. Brain Research, 43, 53.

Goldman-RAKIC, P. S. (1987). Circuitry of the prefrontal cortex and the regulation of behavior by representational memory. In V. B. Mountcastle, F. Plum, \& S. R. Geiger (Eds.), Handbook of neurobiology (pp. 373-417). Bethesda, MD: American Physiological Society.

GoldMAN-RAKIC, P. S. (1990). Cellular and circuit basis of working memory in prefrontal cortex of nonhuman primates. In H. B. M. Uylings, C. G. V. Eden, J. P. C. DeBruin, M. A. Corner, \& M. G. P. Feenstra (Eds.), Progress in brain research (Vol. 85, pp. 325-336). Amsterdam: Elsevier.

Graziano, M. S. A., Yap, G. S., \& Gross, C. G. (1994). Coding of visual space by premotor neurons. Science, 266, 1054-1057.

Hale, S., Myerson, J., Rhee, S. H., Weiss, C. S., \& Abrams, R. A. (1996). Selective interference with the maintenance of location information in working memory. Neuropsychology, 10, 228-240.

Kesner, R. P., Bolland, B. L., \& DaKis, M. (1993). Memory for spatial locations, motor responses, and objects: Triple dissociation among the hippocampus, caudate nucleus, and extrastriate visual cortex. Experimental Brain Research, 93, 462-470.

Lawrence, B. M., Myerson, J., Oonk, H. M., \& Abrams, R. A. (2001). The effects of eye and limb movements on working memory. Memory, 9, 433-444.

Leung, H.-C., Gore, J. C., \& Goldman-Rakic, P. S. (2002). Sustained mnemonic response in the human middle frontal gyrus during on-line storage of spatial memoranda. Journal of Cognitive Neuroscience, 14, 659-671.

Levy, R., Friedman, H. R., Davachi, L., \& Goldman-Rakic, P. S. (1997). Differential activation of the caudate nucleus in primates performing spatial and nonspatial working memory tasks. Journal of Neuroscience, 17, 3870-3882.

LogIE, R. H. (1995). Visuo-spatial working memory. Hove, U.K.: Erlbaum. Logie, R. H., \& MARchetti, C. (1991). Visuo-spatial working memory: Visual, spatial or central executive? In R. H. Logie \& M. Denis (Eds.), Mental images in human cognition (pp. 105-115). Amsterdam: Elsevier.

Mai, J. K., Assheuer, J., \& Paxinos, G. (1997). Atlas of the human Brain. San Diego: Academic Press.

Owen, A. M., Iddon, J. L., Hodges, J. R., Summers, B. A., \& Robbins, T. W. (1997). Spatial and non-spatial working memory at different stages of Parkinson's disease. Neuropsychologia, 35, 519-532.

PoHL, W. (1973). Dissociation of spatial discrimination deficits following frontal and parietal lesions in monkeys. Journal of Comparative \& Physiological Psychology, 82, 227-239.

Postle, B. R., Berger, J. S., TAich, A. M., \& D’Esposito, M. (2000). Activity in human frontal cortex associated with spatial working memory and saccadic behavior. Journal of Cognitive Neuroscience, 12 (Suppl. 2), 2-14.

Postle, B. R., \& D'Esposito, M. (1999a). Dissociation of caudate nucleus activity in spatial and nonspatial working memory: An eventrelated fMRI study. Cognitive Brain Research, 8, 107-115.

Post Le, B. R., \& D'Esposito, M. (1999b). "What"-then-"where" in visual working memory: An event-related f MRI study. Journal of Cognitive Neuroscience, 11, 585-597.

Postle, B. R. \& D'Esposito, M. (2000). Evaluating models of the topographical organization of working memory function in frontal cortex with event-related fMRI. Psychobiology, 28, 132-145.

Postle, B. R., Jonides, J., Smith, E., Corkin, S., \& Growdon, J. H. (1997). Spatial, but not object, delayed response is impaired in early Parkinson's disease. Neuropsychology, 11, 1-9. 
Postle, B. R., Locascio, J. J., Corkin, S., \& Growdon, J. H. (1997). The time course of spatial and object visual learning in early Parkinson's disease. Neuropsychologia, 35, 1413-1422.

Postle, B. R., Stern, C. E., Rosen, B. R., \& Corkin, S. (2000). An f MRI investigation of cortical contributions to spatial and nonspatial visual working memory. NeuroImage, 11, 409-423.

Postle, B. R., Zarahn, E., \& D'Esposito, M. (2000). Using eventrelated fMRI to assess delay-period activity during performance of spatial and nonspatial working memory tasks. Brain Research Protocols, 5, 57-66.

Potegal, M. (1982). Vestibular and neostriatal contributions to spatial orientation. In M. Potegal (Ed.), Spatial abilities, development and physiological foundation (pp. 361-387). New York: Academic Press.

Quinn, J. G., \& Ralston, G. E. (1986). Movement and attention in visual working memory. Quarterly Journal of Experimental Psychology, 38A, 689-703.

RAFAL, R. \& ROBERTSON, L. (1995). The neurology of visual attention. In M. S. Gazzaniga (Ed.), The cognitive neurosciences (pp. 625-648). Cambridge, MA: MIT Press.

RaJKowsKa, G., \& Goldman-Rakic, P. S. (1995). Cytoarchitectonic definition of prefrontal areas in the normal human cortex: II. Variability in locations of areas 9 and 46 and relationship to the Talairach coordinate system. Cerebral Cortex, 5, 323-337.

Rosvold, H. E., \& Delgado, J. M. R. (1956). The effect on delayedattention test performance of stimulating or destroying electrically structures within the frontal lobes of the monkey's brain. Journal of Comparative \& Physiological Psychology, 49, 365-372.

SAlway, A. F. S., \& LogIE, R. H. (1995). Visuospatial working memory, movement control and executive demands. British Journal of Psychology, 86, 253-269.

Semmes, J., Weinstein, S., Ghent, L., \& Teuber, H.-L. (1963). Correlates of impaired orientation in personal and extrapersonal space. Brain, 86, 747-772.

Smith, E. E, Jonides, J., Koepre, R. A., Awh, E., Schumacher, E. H., \& Minoshima, S. (1995). Spatial vs. object working memory: PET investigations. Journal of Cognitive Neuroscience, 7, 337-356.

Smyth, M. M., Pearson, N. A., \& Pendleton, L. R. (1988). Movement and working memory: Patterns and positions in space. Quarterly Journal of Experimental Psychology, 40A, 497-514.

Snyder, L. H., Grieve, K. L., Brotchie, P., \& Andersen, R. A.
(1998). Separate body- and world-referenced representations of visual space in parietal cortex. Nature, 394, 887-891.

TAlairach, J., \& Tournoux, P. (1988). Co-planar stereotaxic atlas of the human brain. New York: Thieme.

Tresch, M. C., Sinnamon, H. M., \& Seamon, J. G. (1993). Double dissociation of spatial and object visual memory: Evidence from selective interference in intact human subjects. Neuropsychologia, 31, 211219.

Ungerleider,L. G., \& Haxby, J. V. (1994). "What" and "where' in the human brain. Current Opinion in Neurobiology, 4, 157-165.

Ungerleider, L. G., \& Mishrin, M. (1982). Two cortical visual systems. In D. J. Ingle, M. A. Goodale, \& R. J. W. Mansfield (Eds.), Analysis of visual behavior (pp. 549-586). Cambridge, MA: MIT Press.

Vallar, G., Lobel, E., Galati, G., Berthoz, A., Pizzamiglio, L., \& Le BiHAN, D. (1999). A fronto-parietal system for computing the egocentric spatial frame of reference in humans. Experimental Brain Research, 124, 281-286.

Woodin, M. E., \& Allport, A. (1999). Independent reference frames in human spatial memory: Body-centered and environment-centered coding in near and far space. Memory \& Cognition, 26, 1109-1116.

Worsley, K. J., \& Friston, K. J. (1995). Analysis of f MRI time-series revisited-again. NeuroImage, 2, 173-182.

Zarahn, E., Aguirre, G. K., \& D'Esposito, M. (1997). A trial-based experimental design for f MRI. NeuroImage, 6, 122-138.

Zarahn, E., Aguirre, G. K., \& D'Esposito, M. (1999). Temporal isolation of the neural correlates of spatial mnemonic processing with functional MRI. Cognitive Brain Research, 7, 255-268.

\section{NOTE}

1. It has recently come to our attention that a model similar to the prospective motor coding model has been described in C. J. Ketcham, T. L. Hodgson, C. Kennard, \& G. E. Stelmach (2003). Memory-motor transformations are impaired in Parkinson's disease. Experimental Brain Research, 149, 30-39.

(Manuscript received November 21, 2002; revision accepted for publication April 18, 2003.) 